\title{
DOCKING STUDIES IN TARGET PROTEINS INVOLVED IN ANTIBACTERIAL ACTION MECHANISMS: ALKALOIDS ISOLATED FROM SCUTELLARIA GENUS
}

\author{
SRI DHARANI R, RANJITHA R, SRIPATHI R, ALI MUHAMMAD KS, RAVI S*
}

Department of Chemistry, Karpagam University, Coimbatore, Tamil Nadu, India. Email: ravisubban@rediffmail.com

Received: 09 May 2016, Revised and Accepted: 18 May 2016

\section{ABSTRACT}

Objective: In the present work, docking study was performed for 22 selected alkaloids isolated from the genus Scutellaria to evaluate their affinity to bacterial proteins that are known targets for many antibiotics with a different mechanism of action: Inhibitors of cell wall synthesis, inhibitors of nucleic acids synthesis and antimetabolites.

Methods: Molecular docking study was carried out using AutoDock 4.2 version and the visualization result using Chimera 1.10 and Discovery Studio 4.5

Result: Among the 22 alkaloids studied, with the DNA gyrase protein 1KZN and a dihydropteroate synthase enzyme 3TYE, the compound scutebarbatine E showed a docking score of -8.5 and $-8.7 \mathrm{Kcal} / \mathrm{mol}$, respectively, involving with hydrophilic and hydrophobic interactions. With respect to $\operatorname{MurD}$ ligase involved in cell wall synthesis $1 \mathrm{UAG}$ and 2X50, the compound 6,7,nicotinyl scutebarbatine G fared well with a docking score of -10.1 and $-10.2 \mathrm{Kcal} / \mathrm{mol}$, respectively. Scutebarbatine G performed well with respect to 3 UDI with binding scores of $-9.3 \mathrm{~K}$ cal/mol.

Conclusion: Overall, it seems that for the selected alkaloids from the genus Scutellaria, the main mechanism of the action is the inhibition of cell wall synthesis.

Keywords: Scutebarbatine, Alkaloids, Molecular docking, Antimicrobial studies.

(C) 2016 The Authors. Published by Innovare Academic Sciences Pvt Ltd. This is an open access article under the CC BY license (http://creativecommons. org/licenses/by/4. 0/) DOI: http://dx.doi.org/10.22159/ajpcr.2016.v9i5.12693

\section{INTRODUCTION}

The classification of antibiotics is based on their mechanism of action, and the main groups include inhibitors of cell wall synthesis, inhibitors of nucleic acids synthesis and antimetabolites [1]. In general, antibiotics inhibit these routes by interacting with specific cell proteins, usually responsible for defined activity. Antimicrobials acting at the cell wall level are the most selective, being bactericidal and presenting a high therapeutic index since inhibition of peptidoglycan synthesis leads to cell lysis [2]. There is a large diversity of antibiotics that can act in different phases of peptidoglycan biosynthesis, namely, in the cytoplasmic, membrane, and parietal phases [3]. $\beta$-lactams act entirely outside the cell membrane, in the final (parietal) phase of peptidoglycan biosynthesis $[3,4]$. They act in penicillin-binding proteins (PBPs), which are responsible for transpeptidation, transglucosylation, and carboxypeptidation reactions. These antibiotics have $\beta$-lactam rings with spatial structures similar to that of the acyl-D-alanyl-D-alanine residues in peptidoglycan chains (natural ligand of PBPs) that link with and inhibit those proteins [3].

Fosfomycin, D-cycloserine, and other antibiotics such as glycopeptides (e.g., vancomycin) and bacitracin, interface between cytoplasmic membrane and cell wall. Some antibiotics such as macrolides, chloramphenicol, lincosamides, streptogramins, and oxazolidinones affect bacteria ribosomes, namely, $30 \mathrm{~S}$ and/or $50 \mathrm{~S}$ subunits, inhibiting the protein synthesis at the initial phases or inducing the synthesis of abnormal proteins [5,6]. Quinolones interfere in DNA replication by inhibiting the activity of bacteria Type II topoisomerase (DNA gyrase) and Type IV topoisomerase [7,8]. Rifampicin inhibits RNA polymerase activity by linking to $\beta$-subunit, preventing the synthesis of mRNA [2]. Folic acid is essential for the synthesis of nitrogen base purines and pyrimidines, and consequently, for the synthesis of DNA. Antimetabolites such as sulfonamides, dapsone, and $p$-aminosalicylic acid act synergistically in two different points of folic acid formation, exhibiting bacteriostatic activity $[2,9,10]$.
Scutellaria (Lamiaceae) includes about 350 species commonly known as skullcaps (Willis, 1966). The genus is widespread and has been widely used in local medicine for thousands of years (Jiangsu New Medical College, 1977). Modern pharmacology research has confirmed that the extracts or monomeric compounds of the genus Scutellaria posses anti-tumor, hepatoprotective, antioxidant, anti-inflammatory, anticonvulsant, antibacterial [10,11], and antiviral effects. Phenolic and terpene compounds, alkaloids, phytosterols, and polysaccharides are the major constituents present in Scutellaria genus. Alkaloids have been isolated mainly from two species Scutellaria barbata (1-11) and Scutellaria flavescens (12-22).

Considering the current increase of antibiotic resistance, the identification of new natural compounds that can be used to treat infections with lower secondary effects than existing antibiotics is becoming crucial to guarantee the health of future generations [12] In this regard, the alkaloids from Scutellaria genus have proved to be particularly interesting sources of bioactive compounds although their mechanisms of action are not yet fully described. Herein, we intended to extend the knowledge on target proteins of standard antibiotics to the antimicrobial alkaloids from Scutellaria genus to predict possible interactions between the alkaloids and target proteins that would allow understanding and describing the mechanism of action. Therefore, docking studies were performed for 22 antimicrobial compounds (Fig. 1) to evaluate their affinity to bacterial proteins that are known targets for some antibiotics.

\section{METHODS}

\section{Experimental}

\section{Molecular docking studies}

Molecular docking studies have been carried out using the AutoDock Tools (ADT) version 1.5.6 and AutoDock version 4.2.5.1 docking program. 


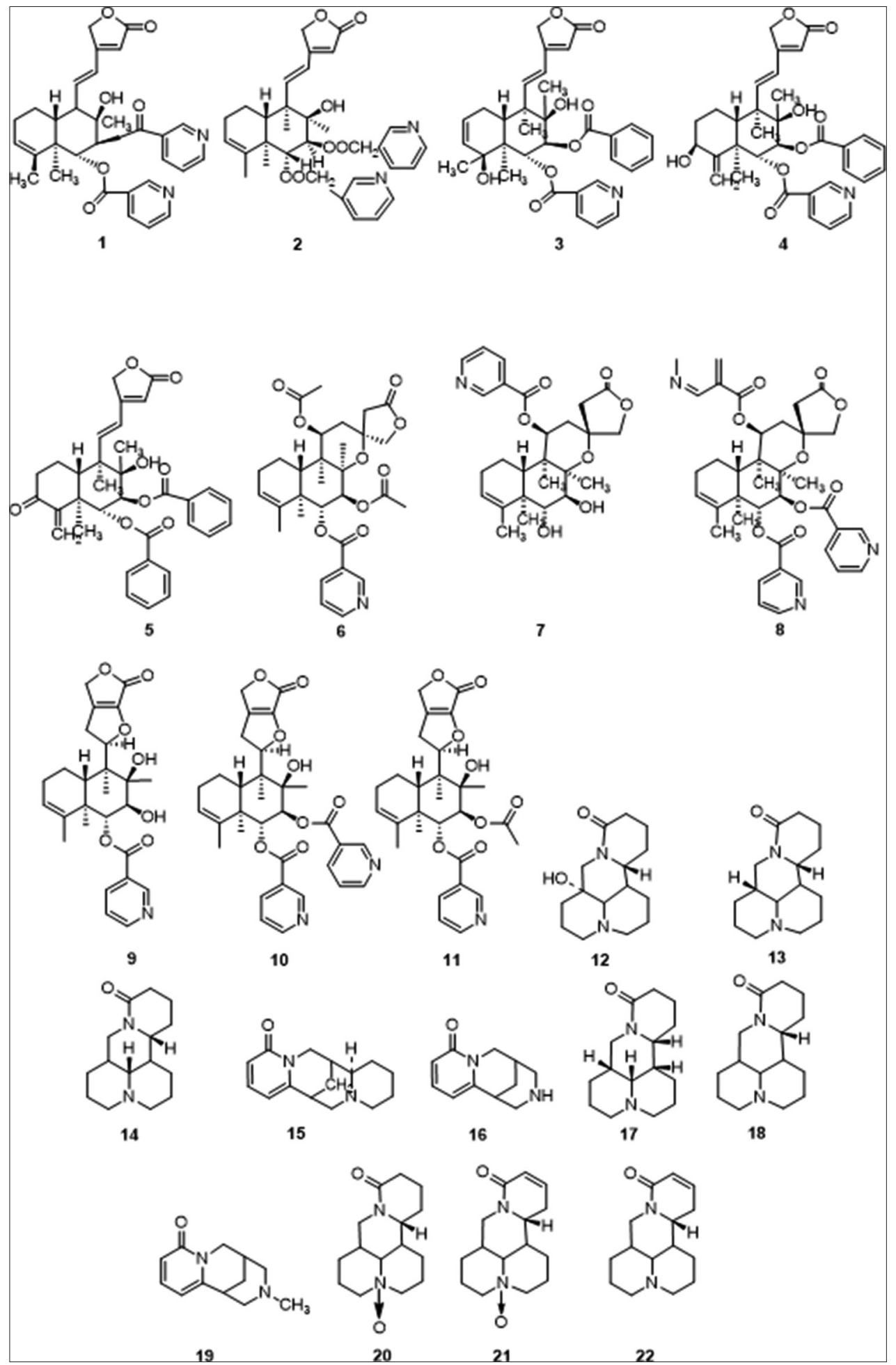

Fig. 1: Chemical structures of the alkaloids subjected for docking studies against antibacterial target proteins

Protein preparation

Target proteins were downloaded from Protein Data Bank (PDB ID:1KZN, 1UAG, 2X50, 3TYE, and 3UDI).

\section{Ligand preparation}

Ligand two-dimensional (2D) structures were drawn using ChemDraw Ultra 8.0 (ChemOffice 2002). Chem3D Ultra 8.0 was used to convert the 2D structure into three-dimensional (3D), and the energy minimized using semi-empirical AM1 method. Minimize energy to the minimum root mean square gradient of 0.100 was set in each iteration. All structures were saved as pdb file format for input to ADT. All the ligand structures were then saved in SDF file format, to carry out docking in Autodock Vina.

\section{Grid formation}

A grid box with dimension of $40 \times 40 \times 40 \mathrm{~A}^{3}$ with $0.375 \mathrm{~A}$ spacing and centered on $29.470,47.997$, and 8.863 was created around the binding site of protein using ADT. The center of the box was set at ligand center, and grid energy calculations were carried out. 
Table 1: Docking scores (kcal/mol) of the 22 alkaloids with the five antibacterial proteins

\begin{tabular}{|c|c|c|c|c|c|c|}
\hline Ligand & Number & $1 \mathrm{KZN}^{\mathrm{a}}$ & 1UAG ${ }^{b}$ & $2 \times 50^{b}$ & 3UDI ${ }^{b}$ & 3 TYE $^{\mathrm{c}}$ \\
\hline Scutebarbatine A & 1 & -7.8 & -8.8 & -8.1 & -7.7 & -8.2 \\
\hline Scutebarbatine B & 2 & -8.0 & -8.2 & -8.1 & -7.9 & - \\
\hline Scutebarbatine C & 3 & -8.4 & -9.1 & -8.6 & -8.5 & -8.6 \\
\hline Scutebarbatine D & 4 & -7.4 & -8.8 & -8.2 & -8.0 & -8.5 \\
\hline Scutebarbatine E & 5 & -8.5 & -9.2 & -8.4 & -8.7 & -8.7 \\
\hline Scutebarbatine F & 6 & -7.4 & -8.6 & -8.0 & -7.8 & -7.6 \\
\hline Scutebarbatine G & 7 & -8.2 & -9.2 & -8.8 & -9.3 & -8.5 \\
\hline 6, 7-0-nicotinyl scutebarbatine G & 9 & -8.3 & -10.1 & -10.2 & -8.6 & -8.5 \\
\hline 6-0-nicotinyl-0-acetyl scutebarbatine G & 10 & -7.8 & -9.5 & -8.7 & -8.2 & -7.7 \\
\hline 7-0-nicotinyl scutebarbatine $\mathrm{H}$ & 11 & -7.7 & -9.4 & -8.4 & -8.5 & -8.3 \\
\hline Sopharnol & 12 & -7.1 & -7.1 & -6.8 & -7.4 & -7.1 \\
\hline Sophoridine & 13 & -7.5 & -7.1 & -6.7 & -7.4 & -6.6 \\
\hline Allmatrine & 14 & -6.5 & -6.2 & -5.9 & -7.0 & -6.1 \\
\hline Anagyrine & 15 & -6.7 & -7.0 & -6.8 & -7.6 & -7.4 \\
\hline Cytosine & 16 & -6.2 & -7.1 & -6.7 & -7.2 & -6.8 \\
\hline $\mathrm{N}$-methyl cytosine & 19 & -7.4 & -7.0 & -7.4 & -7.7 & - \\
\hline Oxymatrine & 20 & -7.0 & -7.2 & -7.3 & -7.6 & -6.1 \\
\hline Osysocarpine & 21 & -6.2 & -6.9 & -6.8 & -.4 & -6.2 \\
\hline Sophocarpine & 22 & -6.2 & -5.9 & -5.7 & -6.0 & -6.7 \\
\hline
\end{tabular}

${ }^{\mathrm{a}}$ Inhibitors of nucleic acid synthesis, ${ }^{\mathrm{b}}$ Inhibitors of cell wall synthesis, ${ }^{\mathrm{c}}$ Antimetabolites inhibitor

\section{Docking protocol}

For the AutoDock docking calculation, default parameters were used and 10 docked conformations were generated for each compound. The energy calculations were done using genetic algorithms. The outputs were exported to Chimera 1.10 and Discovery Studio 4.5 for visual inspection of the binding modes and interactions of the compounds with amino acid residues in the active site [13].

\section{RESULT AND DISCUSSION}

A docking study of target proteins involved in antibacterial mechanisms was performed to extend the knowledge on standard antibiotics to alkaloids from Scutellaria genus with reported antibacterial activity. The proteins used were the following: A DNA-Gyrase (PDB id 1KZN), an enzyme MurD ligase (PDB id 1UAG, 2X50, and 3UDI) involved in cell wall synthesis and dihydropteroate synthase enzyme (DHPS; PDB id 3TYE). To validate the docking approach for the protein structures used, the respective ligands (alkaloids) were docked to the active site of each protein using AutoDock4. Some protein structures presented natural substrates as a co-crystallized ligand, whereas in others the co-crystallized ligand was a known inhibitor, in both cases the same docking and scoring validation process were used. Each co-crystalized ligand was previously removed from the respective protein binding site. The predicted docking pose was compared with the experimental cocrystallized binding pose (Table 1).

The results are presented in Table 1 . The docking score, hydrogen bonded residues, and hydrophobic interactions such as alkyl and pi alkyl, Vander Waals interactions were provided. Most of the alkaloids showed very good interactions with the studied proteins. Among the 22 alkaloids studied, with the protein 1KZN and 3TYE, the compound 5 showed a docking score of -8.5 and $-8.7 \mathrm{Kcal} / \mathrm{mol}$ with hydrophilic and hydrophobic interactions. With respect to 1 UGAG and 2X50, the compound 9 fared well with a docking score of -10.1 and $-10.2 \mathrm{Kcal} / \mathrm{mol}$. Compound 7 performed well with respect to 3UDI with binding scores of $-9.3 \mathrm{~K} \mathrm{cal} / \mathrm{mol}$ (Fig. 2).

Molecular docking was carried out to determine the binding mode of the inhibitor within the MurD active site. Here, the inhibitor moiety occupies the same site as the D-Glu residue of the product UMAG of the enzymatic reaction catalyzed by MurD. The benzene ring of the compound 9 with 1 UAG protein forms a pi-alkyl interaction with Lys348 and had Van Der Waals interaction with Thr321. The carbonyl group of the $\alpha, \beta$-unsaturated- $\gamma$-lactone moiety forms hydrogen bonds with Ser 415 and Phe 422 . Furthermore, the oxygen atom of the lactone moiety forms hydrogen bond with Leu416. The carbonyl group of the benzoyl group forms a hydrogen bond with His183. Further, the molecule is well placed within the hydrophobic pocket which consists of the amino acids Gly73, Asn138, Phe161, Arg37, Thr16, and Ser71. The interactions are similar to the interactions present in MurD-(R)-32 complex reported by Zidar et al. [14]. With 1 UAG protein, the carbonyl group of the $\alpha, \beta$ unsaturated- $\gamma$-lactone moiety of 4 showed hydrogen bonds with the key amino acids Lys 348 and Phe422 exhibiting a docking score of -8.8 $\mathrm{Kcal} / \mathrm{mol}$. Compound 5 forms hydrogen bonds with the Ser415, Arg302, Lys319, and Leu416 and exhibited a score of $-9.2 \mathrm{Kcal} / \mathrm{mol}$. Similarly, compound 6 and 8 also forms hydrogen bonds with Ser415, Arg37, Gly73, and Leu416. The hydrogen bonds are due to the carbonyl group and the oxygen atom of the $\alpha, \beta$-unsaturated- $\gamma$-lactone moiety. The alkaloids reported from the $S$. barbata [1-11] exhibited comparatively higher scores than the alkaloids reported from $S$. flavescens (12-22). This may be attributed to the reason that the alkaloids from $S$. barbata contain $\alpha, \beta$-unsaturated- $\gamma$-lactone moiety to form hydrogen bonds and aromatic rings and alkyl group for hydrophobic interactions.

Furthermore, compound 9 with 2X50 protein forms hydrogen bond with Thr16, Leu15, and a good number of Van der Waals interaction was present. In compounds 4 and 5 , the oxygen and the carbonyl group of the $\alpha, \beta$-unsaturated- $\gamma$-lactone moiety forms hydrogen bonds with the key residues Ser415, Thr16, and Leu13.

Compound 5 was the best-scored compound for the protein 3UDI. This compound interacts with 3UDI by forming hydrogen bonds with Gly709 and Thr672, Thr654, Ser470, and Arg486 explaining the high affinity for this protein and suggesting a possible mechanism of the action. All the above three proteins prevent the cell wall synthesis by inhibiting MurD. So, all the alkaloids reported from the $S$. barbata which contains the $\alpha, \beta$-unsaturated- $\gamma$-lactone moiety act as very good MurD inhibitors and prevents the cell wall synthesis.

Further with the DHPS, compound 5 showed a high docking score of $-8.7 \mathrm{Kcal} / \mathrm{mol}$. The oxygen and the carbonyl group of the $\alpha, \beta$-unsaturated- $\gamma$-lactone moiety form hydrogen bonds with Ser221 and Phe222, respectively. Furthermore, the hydroxyl group presents in compound 5 forms hydrogen bond with Ala190 and the NH group forms a hydrogen bond with Asp149. Further, it forms hydrophobic interaction with Met151. 


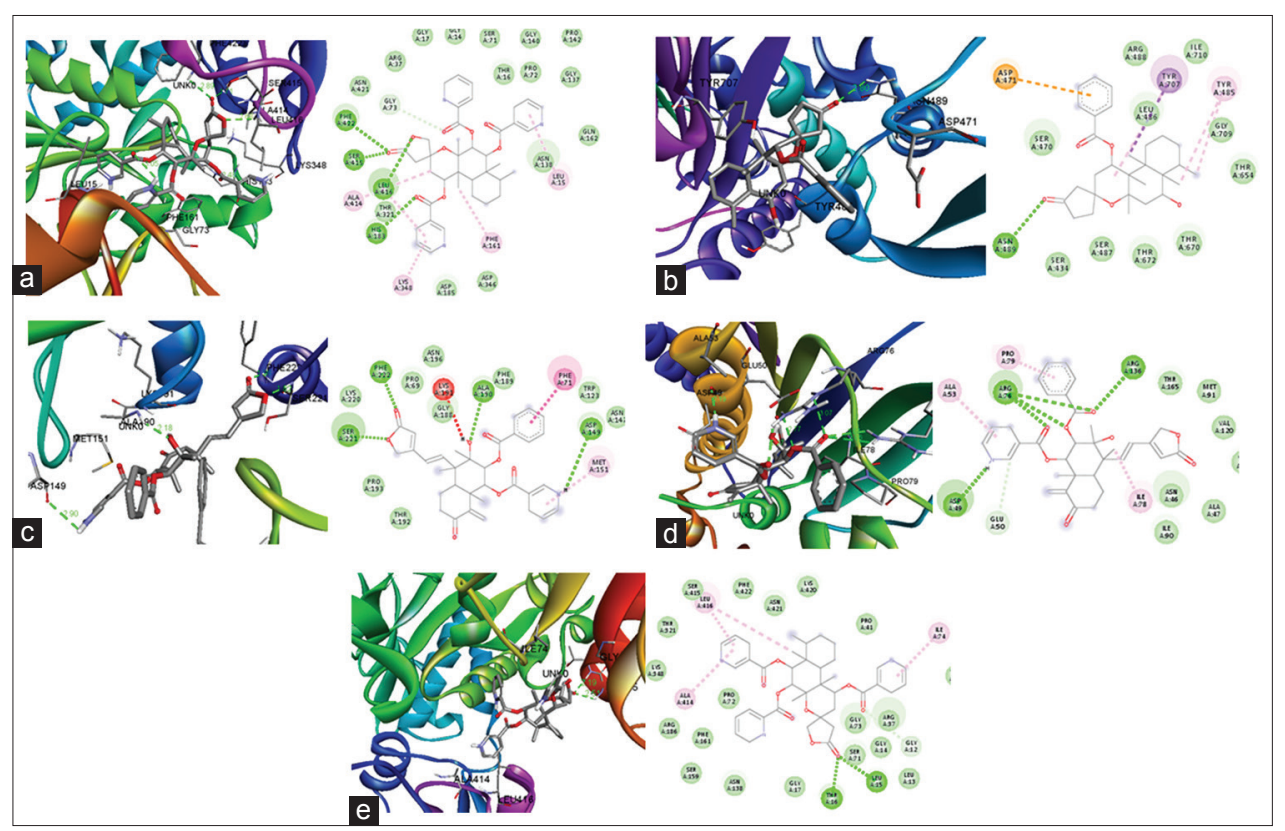

Fig. 2: Three-dimensional (3D) and two-dimensional (2D) binding interaction of best scoring compound with different protein, (a) 3D and 2D binding interaction 9 with MurD protein (PDB ID:1UAG), (b) 3D and 2D binding interaction 7 with Escherichia coli protein (PDB ID:3UDI), (c) 3D and 2D binding interaction 5 with $E$. coli protein (PDB ID:3TYE), (d) 3D and 2D binding interaction 5 with $E$. coli protein (PDB ID:1KZN), (e) 3D and 2D binding interaction 9 with MurD protein (PDB ID:2X50)

The docking results obtained from AutoDock showed that the studied compounds can be accommodated in the binding pocket of BaDHPS with a comparable orientation to the one observed in the streptozotocin (STZ)-dihydropterin pyrophosphate (DHPP) covalent adduct in the reported crystal structure18. The top-ranked docking poses reproduce the key interactions observed in the STZ-DHPP-BaDHPS complex (Fig. 2a). Most notably, the $\alpha, \beta$-unsaturated- $\gamma$-lactone moiety in all predicted binding modes interacts with Ser221 via H-bonding between its oxygen atom and the backbone $\mathrm{NH}$ group, while the phenyl ring packs against the side chains of Lys220 and Pro69. Moreover, the central phenyl moiety in the studied compounds makes face to edge interaction with Phe189. As shown in Fig. 2, this characteristic orientation of the $\alpha, \beta$-unsaturated- $\gamma$-lactone is common among all studied compounds indicating that all of them can occupy the paraaminobenzoic acid (PABA) pocket preventing the key substrate from binding, which is the basis of the inhibitory action of all sulfa drugs against DHPS [15-17].

In addition to the distinctive interactions in the PABA-binding pockets, the studied compounds exhibit interactions with residues in the pterinbinding pocket of DHPS that vary slightly between compounds. For instance, the compound 9 exhibits cation - interaction between its phenyl ring and the charged side chain of Lys220, as well as a hydrogen bond with the side chain of Arg254, which could further stabilize its interaction with DHPS

In the present investigation, an attempt was made to understand the ligand-receptor interactions of the alkaloids against bacterial DNA gyrase as a target enzyme. For scute barbatine E, the obtained pose showed four hydrogen-bond interactions with Arg76, Arg36, and Asp49. It also showed hydrophobic interactions with Ala53, Pro79, and Ile78. And exhibited the highest score of $-8.5 \mathrm{Kcal} / \mathrm{mol}$ among the tested compounds. All the other compounds also showed similar interactions.

Molecular docking studies showed that the synthesized compounds could act as inhibitors for the DHPS, DNA gyrase, and MurD ligase. This study is a platform for the future design of more potent antimicrobial agents. Moreover, docking studies indicated that the target compounds could occupy both the PABA and pterin-binding pockets of DHPS.

\section{CONCLUSION}

The data presented herein highlighted some conclusions regarding the affinity of different mushroom compounds to protein targets related to the antibacterial action. However, several compounds indicated that main mechanism of their action is the inhibition of cell wall synthesis, being MurD is a probable protein target. However, some relevant affinities of compounds were observed in the docking study, which could indicate possible mechanisms.

\section{ACKNOWLEDGMENT}

We express our gratitude to Management Karpagam University, Coimbatore - 21, for providing the laboratory facilities and encouragement.

\section{REFERENCES}

1. Tenover FC. Mechanisms of antimicrobial resistance in bacteria. Am J Med 2006;119 6 Suppl 1:S3-10.

2. Murray PR, Rosenthal KS, Pfaller MA. Microbiologia Médica. $5^{\text {th }}$ ed. Rio de Janeiro, Brasil: Mosby, Elsevier; 2006.

3. Calvo J, Martínez-Martínez L. Mecanismos de accion de los antimicrobianos. Enferm Infecc Microbiol Clin 2009;27:44-52.

4. Scholar EM, Pratt WB. The inhibitors of cell wall synthesis, I. Mechanism of action of the penicillins, cephalosporins, vancomycin, and other inhibitors of cell wall synthesis. In: Scholar EM, Pratt WB editors. The Antimicrobial Drugs. Oxford, UK: Oxford University Press; 2000. p. 51-80

5. Kohanski MA, Dwyer DJ, Collins JJ. How antibiotics kill bacteria: From targets to networks. Nat Rev Microbiol 2010;8(6):423-35.

6. Kostopoulou, ON, Kourelis TG, Mamos P, Magoulas GE, Kalpaxis DL. Insights into the chloramphenicol inhibition effect on peptidyl transferase. Activity, using two new analogs of the drug. Open Enzyme Inhib J 2009;4:1-10.

7. Golan DE. Princípios de Farmacologia: A Base Fisiopatológica da Farmacoterapia. $2^{\text {nd }}$ ed. Rio de Janeiro, Brasil: Guanabara Koogan; 2009. p. $547-61$.

8. Brugueras M, Garcia M, Diaz R. Actualidad de las quinolonas. Rev Cubana Farm 2005;39(1):1-15.

9. Huang L, Crothers K, Atzori C, Benfield T, Miller R, Rabodonirina M, et al. Dihydropteroate synthase gene mutations in Pneumocystis and sulfa resistance. Emerg Infect Dis 2004;10(10):1721-8.

10. Alves MJ, Ferreira IC, Dias J, Teixeira V, Martins A, Pintado M. 
A review on antimicrobial activity of mushroom (Basidiomycetes) extracts and isolated compounds. Planta Med 2012;78(16):1707-18.

11. Alves MJ, Ferreira IC, Froufe HJ, Abreu RM, Martins A, Pintado M Antimicrobial activity of phenolic compounds identified in wild mushrooms, SAR analysis and docking studies. J Appl Microbiol 2013;115(2):346-57.

12. Radulovic NS, Blagojevic PD, Stojanovic-Radic ZZ, Stojanovic NM Antimicrobial plant metabolites: Structural diversity and mechanism of action. Curr Med Chem 2013;20(7):932-52.

13. Trott O, Olson AJ. AutoDock Vina: Improving the speed and accuracy of docking with a new scoring function, efficient optimization, and multithreading. J Comput Chem 2010;31(2):455-61.

14. Zidar N, Tomasic T, Sink R, Rupnik V, Kovac A, Turk S, et al. Discovery of novel 5-benzylidenerhodanine and 5-benzylidenethiazolidine-2,4 dione inhibitors of MurD ligase. J Med Chem 2010;53(18):6584-94.

15. Yun MK, Wu Y, Li Z, Zhao Y, Waddell MB, Ferreira AM, et al. Catalysis and sulfa drug resistance in dihydropteroate synthase. Science 2012;335(6072):1110-4

16. Bock L, Miller GH, Schaper KJ, Seydel JK. Sulfonamide structure-activity relations in a cell-free system. 2. Proof for the formation of a sulfonamide-containing folate analog. J Med Chem 1974;17(1):23-8

17. Roland S, Ferone R, Harvey RJ, Styles VL, Morrison RW. The characteristics and significance of sulfonamides as substrates for Escherichia coli dihydropteroate synthase. J Biol Chem 1979;254(20):10337-45. 